\title{
CEADS: UM CENTRO DE PESQUISA DA UNIVERSIDADE DO ESTA- DO DO RIO DE JANEIRO E SUA CONTRIBUIÇÃO PARA A MELHORIA DAS CONDIÇÕES HUMANAS E AMBIENTAIS
}

CEADS: A RESEARCH CENTRE OF THE UNIVERSITY OF RIO DE JANEIRO STATE AND ITS CONTRIBUTES TO THE IMPROVEMENT OF HUMAN AND ENVIRONMENTAL

\begin{abstract}
Autores
Cátia Henriques Callado. Professora Associada da Universidade do Estado do Rio de Janeiro (UERJ), Rio de Janeiro, RJ, Brasil.

E-mail: catia.callado@gmail.com
\end{abstract}

Marcos Bastos. Professor Associado da Universidade do Estado do Rio de Janeiro, Rio de Janeiro (UERJ), RJ, Brasil.

E-mail: mbastosp@gmail.com

Carla Y Gubaú Manão. Bióloga Pesquisadora da Universidade Federal do Rio de Janeiro (UFRJ), Rio de Janeiro, RJ, Brasil.

E-mail: carlaygm@gmail.com

Norma Albarello. Professora Associada da Universidade do Estado do Rio de Janeiro (UERJ), Rio de Janeiro, RJ, Brasil.

E-mail: albarellon@gmail.com

Recebido em: 28/01/2019 Aprovado em: 31/01/2019

DOI: $10.12957 /$ interag.2018.25096

\section{Artigo}

\section{Resumo}

Este artigo analisa o desempenho do Centro de Estudos Ambientais e Desenvolvimento Sustentável (CEADS) em um cenário de produção, inovação, aplicação e colaboração-articulação do conhecimento. O inventário é baseado no banco de dados das atividades do CEADS e no Currículo Lattes dos coordenadores dos projetos do CEADS no Conselho Nacional de Desenvolvimento Científico e Tecnológico. Os resultados mostram que o CEADS cumprem as diretrizes estabelecidas para a criação ou manutenção de um centro de pesquisa universitário, contribuindo para a melhoria das condições humanas e ambientais sem deixar função

\section{Abstract}

This paper analyses the performance of the Centre for Environmental Studies and Sustainable Development (CEADS) in a scenario of production, innovation, application and collaboration-articulation of knowledge. The survey is based on database of CEADS activities and the Lattes Curriculum from the coordinators of CEADS Projects at the National Council for Scientific and Technological Development. The results show that CEADS fulfils the established guidelines for the creation or maintenance of a university research centre. CEADS contributes to the improvement of human and environmental conditions without leaving the 
de ser um espaço participativo de discussão, análise e crítica das questões atuais. Por meio do CEADS, a Universidade do Estado do Rio de Janeiro reforça sua liberdade e autonomia, o que é vital para uma universidade pluricultural, diversificada e sempre revolucionária.

Palavras- chave: Meio ambiente; Desenvolvimento Regional; Rede de Pesquisa; Universidade Pública

Área Temática: Gestão Universitária Linha Temática: Pesquisa; Extensão e Ensino em Meio Ambiente function of being a participatory space for discussion, analysis and critique of current questions. Through CEADS, the University of Rio de Janeiro State reinforces its freedom and autonomy, which is vital for a multicultural, diversified and always revolutionary university.

Keywords: Environment; Regional development; Research Network; Public University

\section{Introdução}

Os centros de pesquisa universitários apresentam um papel importante para o avanço do conhecimento, o estabelecimento de novas políticas, a formação de recursos humanos e a aplicação de seus resultados em favor da sociedade, de forma produtiva e benéfica ${ }^{1,2}$. Esses centros diferem consideravelmente entre si, podendo ser grandes ou pequenos, administrados por uma única instituição ou por um consórcio de instituições diferentes. Entretanto, assemelham-se no fato de apresentar propostas abrangentes e de alcance inter e transdisciplinar, o que aumenta o valor institucional da Universidade que possui esses centros, tanto para estudantes, como para professores, funcionários e sociedade. Assim, um centro de pesquisa pode ser um patrimônio importante para a instituição onde está inserido, que pode trazer prestígio, reconhecimento, apoio e, até mesmo, recursos financeiros. No entanto, os centros de pesquisa universitários não são muito comuns na América Latina ${ }^{3,4}$.

As diretrizes que estabelecem a criação ou a manutenção de um centro de pesquisa universitário são subjetivas e não sistematizadas. De modo geral, um centro de pesquisa deve atender a oito perguntas gerais como estratégia para sua existência:

1 - As ações do centro de pesquisa são criticamente importantes para o sucesso da Universidade?

2 - O centro de pesquisa apresenta ações transformadoras e potencial para ocupar uma posição de liderança entre as instituições pares?

3 - O centro de pesquisa é capaz de levantar fundos para apoiar a si mesmo?

4 - O centro de pesquisa é capaz de contribuir para formação de excelência de alunos?

5 - O centro de pesquisa é capaz de estimular o desenvolvimento de novos currículos?

6 - O centro de pesquisa é capaz de fortalecer a marca da Universidade?

7 - O centro de pesquisa é capaz de influenciar outras instituições e setores além daqueles que participam de sua própria iniciativa? e

8 - O centro de pesquisa exerce impacto sobre o mundo exterior? 
Neste trabalho, essas questões são aplicadas para avaliar o desempenho do Centro de Estudos Ambientais e Desenvolvimento Sustentável (CEADS), Centro de Pesquisa da Universidade do Estado do Rio de Janeiro (UERJ), em um cenário de produção, inovação, aplicação e colaboração/articulação do conhecimento. O CEADS foi inaugurado em 1998 para atender ao compromisso firmado com o governo do estado do Rio de Janeiro, por meio do Decreto no 19.983, que transferiu à UERJ toda a área e benfeitorias ocupadas pelo extinto presídio de segurança máxima: Instituto Penal Cândido Mendes ${ }^{5}$. Este termo previa a implantação de um centro de pesquisa e de um museu voltados à preservação, à divulgação e ao desenvolvimento ambiental e social.

\section{Material e Métodos}

O CEADS está localizado na Ilha Grande, município de Angra dos Reis, no sul do estado

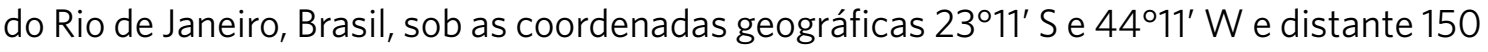
$\mathrm{Km}$ do campus sede da Universidade do Estado do Rio de Janeiro (Figura 1). A região apresenta grande interesse biológico, histórico, social e econômico. A llha faz parte do corredor biológico da Serra do Mar, é indicada como um refúgio pleistocênico, é reconhecida como um núcleo histórico e patrimônio cultural da humanidade e está incluída na Reserva da Biosfera da Mata Atlântica ${ }^{6}$. Na região, também são encontrados: um terminal petrolífero, duas usinas nucleares (Angra I e II), um grande estaleiro, um porto comercial adaptado para serviços off-shore e um terminal de exportação de minério de grande porte. Somam-se, ainda, as atividades de maricultura, pesca e turismo que reunidos representam um dos grandes desafios ao desenvolvimento e à sustentabilidade da região 5 .

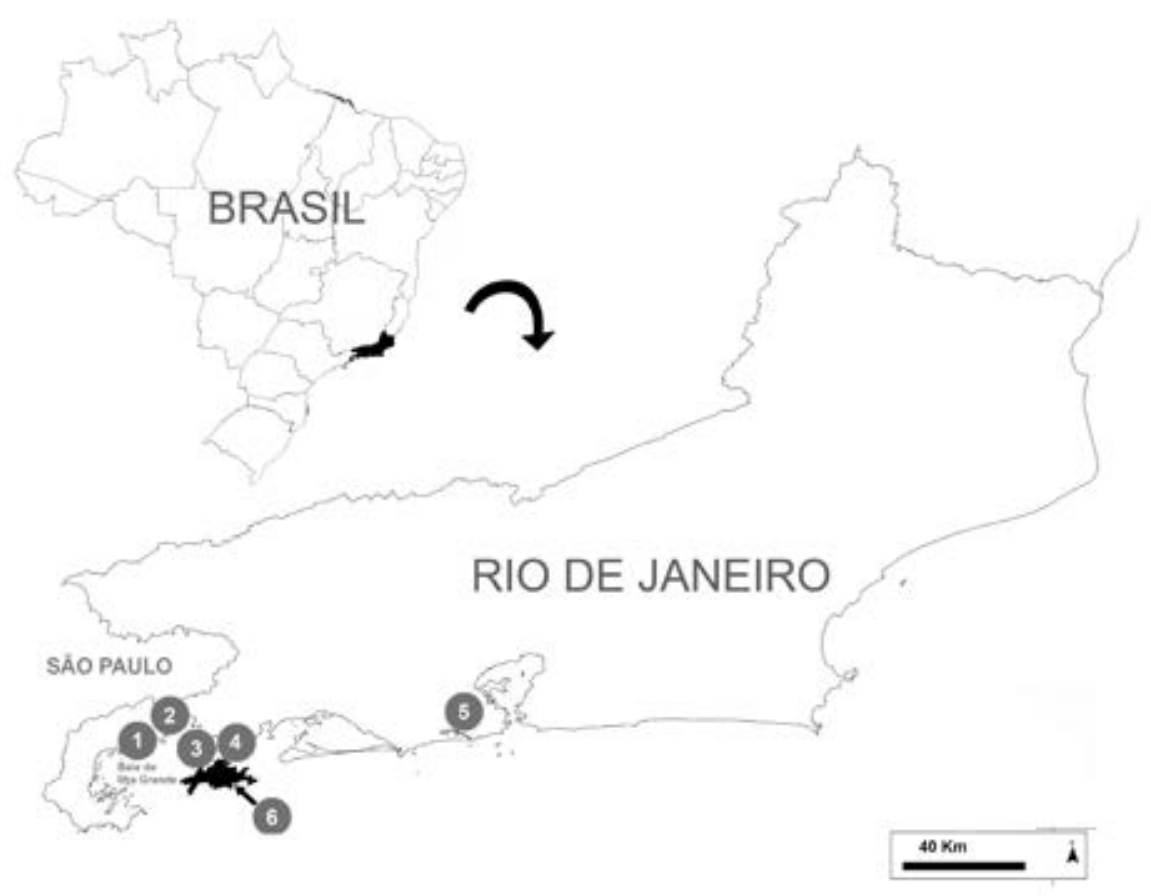

Figura 1. Mapa da Ilha Grande no estado do Rio de Janeiro, Brasil.

1. Central Nuclear de Angra dos Reis (Angra 1, 2 e 3).

2. Aeroporto de Angra dos Reis.

3. Empresa Brasileira de Petróleo (Petrobrás). 
4. Estação de Transporte Aquaviário (CCR Barcas).

5. Campus sede da Universidade do Estado do Rio de Janeiro (UERJ), município do Rio de Janeiro.

6. CEADS

Os dados analisados neste trabalho são referentes ao período de 2006 a 2015 e compreendem: número e tipo de projeto cadastrado no CEADS; número e perfil de usuários em atividade no CEADS; quantidade e tipo de produção científica dos coordenadores de projetos (CP) cadastrados no CEADS; rede de colaboração-articulação dos CPs cadastrados no CEADS; e recursos financeiros externos à UERJ, investidos na infraestrutura geral do CEADS e dos laboratórios associados à captação destes recursos. Os dados foram inventariados a partir dos formulários de cadastro de projetos; dos relatórios anuais dos CPs e de seus currículos disponibilizados na Plataforma Lattes do Conselho Nacional de Desenvolvimento Científico e Tecnológico (www.cnpq.br).

Para avaliação do potencial do CEADS como base para redes em ciência, tecnologia e inovação, foram exemplificados três CPs, de faixa etária semelhante (entre 50 e 55 anos), de nacionalidade brasileira, formação profissional no Brasil, com inserção em cursos de graduação e pós-graduação, de um mesmo Instituto (Is) e considerados de pequena (CP1), média (CP2) e grande (CP3) colaboração-articulação científica com outros pesquisadores e instituições, representados apenas na produção bibliográfica autodeclarada em seus currículos publicados na Plataforma Lattes do Conselho Nacional de Desenvolvimento Científico e Tecnológico. Os níveis hierárquicos de colaboração-articulação inventariados foram com: Pesquisadores do próprio Departamento (D), Alunos de Graduação (G), Alunos de Pós-graduação (P), Pesquisadores do próprio Instituto (Is), Pesquisadores da própria Universidade (U), Pesquisadores de outra Instituição no Rio de Janeiro (RJ), Pesquisadores de outra Instituição no Brasil (B) e Pesquisadores de Instituições de outros Países (It) (Figura 5). Os níveis hierárquicos e respectivos números de colaboração-articulação foram estabelecidos em orbitais plotadas a partir do CP e da distância em relação a sua unidade de lotação acadêmica na Universidade do Estado do Rio de Janeiro. Foram omitidas a identificação dos pesquisadores, de suas unidades acadêmicas na UERJ e das unidades e instituições cujos pesquisadores integram a rede de cada CP exemplificado. Cada colaborador foi computado apenas uma vez no estabelecimento da articulação com o CP amostrado.

\section{Resultados e Discussão}

A Rede Interamericana de Academias de Ciência aponta a pesquisa científica e tecnológica como fundamental ao desenvolvimento integral das sociedades e suas economias, que baseadas no conhecimento podem contribuir, efetivamente, para o crescimento econômico e aumento da produtividade. Ressaltamos, entretanto, que este crescimento somente pode ser considerado integral quando, por meio da pesquisa científica e tecnológica, prevê as melhores práticas de meio ambiente e sustentabilidade.

Segundo Chaimovich ${ }^{7}$, no século XXI, é inconcebível pensar em trabalhos voltados ao fortalecimento da governabilidade democrática e melhoria da qualidade de vida, sem usar a ciência de forma extensiva, aplicar tecnologias adequadas localmente, introduzir o conceito de inovação em todos os níveis da sociedade e melhorar a educação científica. A UNESCO, na Conferência Mundial sobre Ciência de 1999, já postulava que a educa- 
ção científica é um pré-requisito à democracia e ao desenvolvimento das nações ${ }^{8}$. Entre as metas estabelecidas para a ampliação da educação científica, a UNESCO ressaltava a formação direcionada dos professores; a reestruturação dos currículos, metodologias e recursos instrucionais e o investimento em museus e centros de pesquisa. É nesse contexto que apresentamos o CEADS, único centro de pesquisas estruturalmente consolidado como campus avançado da UERJ. A partir da gestão que se iniciou em 2008 , foram implementadas ações que permitiram consolidar o CEADS como um centro de pesquisa de excelência, em sintonia com sua relevância ambiental e regional. Esse resultado foi possível devido à uma política de apoio ao Centro de Pesquisa, com a ampliação de pessoal e investimentos na capacidade instalada para suporte às suas atividades acadêmicas. A criação de uma assessoria e de uma coordenação científica permitiu agregar os pesquisadores atuantes no CEADS, que lideraram programas e projetos para captação de recursos financeiros (Figura 2), em torno de objetivos dirigidos às políticas públicas sociais, econômicas e ambientais. Este resultado se deve à organização de equipes de pesquisa multidisciplinares e interdepartamentais com atuação no CEADS.

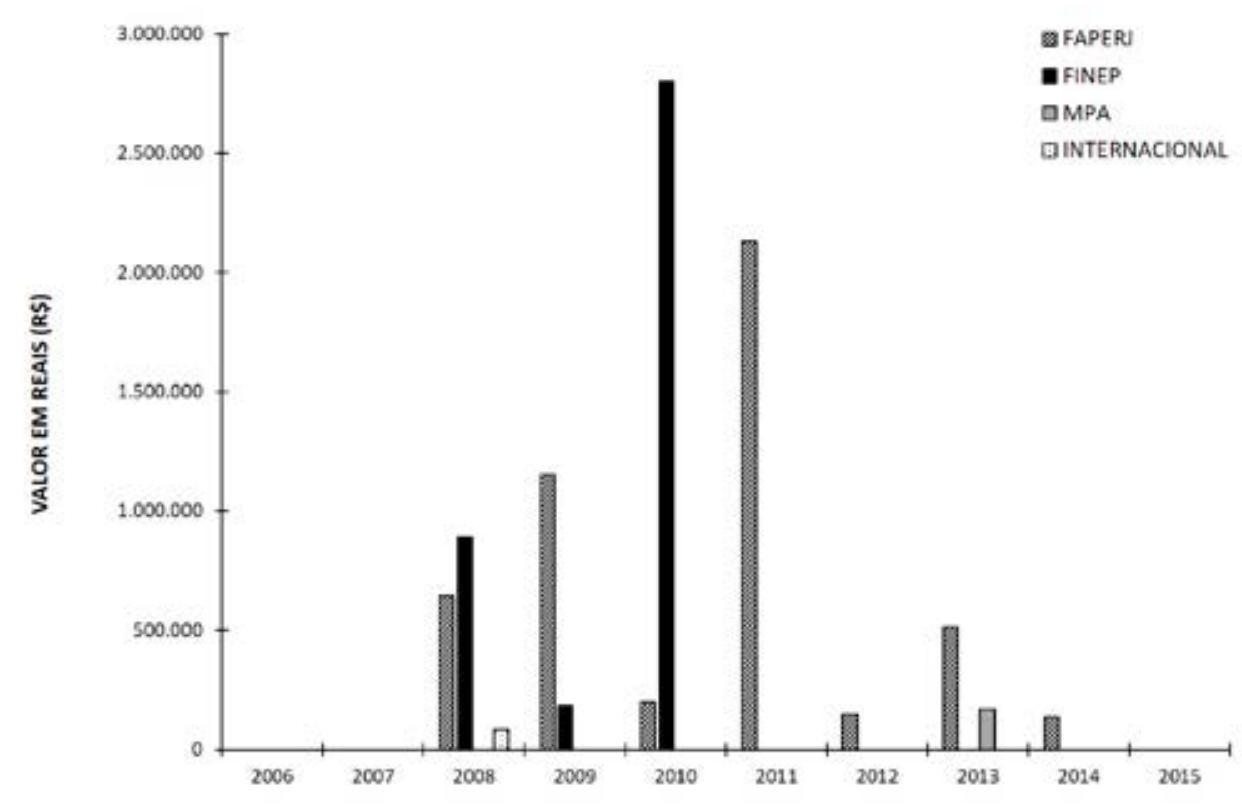

Figura 2. Recursos financeiros externos outorgados ao CEADS por agências de fomento no período de 2006 a 2015. FAPERJ: Fundação Carlos Chagas Filho de Amparo à Pesquisa do Estado do Rio de Janeiro. FINEP: Empresa Brasileira de Inovação e Pesquisa. MPA: Ministério de Pesca e Aquicultura do Brasil. INTERNACIONAL: GITEC Consult GmbH. Os valores apresentados incluíram somente recursos financeiros para o CEADS obtidos por projetos de abrangência geral. Cotação média do dólar no período U\$ $1=\mathrm{R} \$ 3$ - Fonte Reuters).

Na região sul fluminense, o CEADS, no período de 2006 a 2015, também consolidou parceria com Prefeituras e Unidades de Conservação em âmbito estadual e federal, com destaque para sua atuação na elaboração dos estudos ambientais para os Planos Diretores do Parque Estadual da Ilha Grande e para a delimitação das áreas de interesse e 
normas específicas de uso (Zoneamento) de toda Ilha Grande (193 Km²), que apontam esta Unidade de Conservação do Brasil como a que apresenta o mais diverso conhecimento a respeito de seus recursos, incluindo os naturais, sociais, culturais e econômicos. Em 2010, o CEADS atingiu o cenário mundial da conservação ambiental, sendo uma das escalas da expedição oceanográfica internacional: Tara Océans. A expedição francesa, sob os auspícios do Programa das Nações Unidas para o Meio Ambiente (PNUE), visou estudar ecossistemas oceânicos ameaçados por perturbações ecológicas importantes como a mudança climática e a poluição em todos os mares do planeta Terra. Como marco desta expedição, o CEADS foi sede do workshop de cooperação internacional "UERJ-Instituições Francesas: meio ambiente e ciências do mar".

Neste período, o CEADS contribuiu anualmente para a formação contextualizada de aproximadamente 600 alunos (Figura 3). Os alunos em disciplinas representaram o maior número de usuários do CEADS e o conjunto formado pelos professores, técnicos e alunos em atividade de pesquisa somaram $42 \%$ do total de usuários. Esses discentes puderam complementar sua formação vivenciando um conjunto diversificado de experiências reais distribuídas em diferentes disciplinas dividas em nível de graduação e pós-graduação (Figura 4). Os cursos de graduação de Ciências Biológicas, Geografia, História e Oceanografia figuraram entre os que mais realizaram atividades acadêmicas no CEADS. É importante ressaltar que programas de pós-graduação stricto sensu da UERJ, como o de Ecologia e Evolução (PPGEE), o de Biologia Vegetal (PGBV), o de Oceanografia (PPG/ OCN), o de Meio Ambiente (PPMA) e o de Engenharia Ambiental (PEAMB) incluem o CEADS como parte de sua infraestrutura básica. Alguns dos cursos e programas de pós- graduação da UERJ tiveram seus currículos estabelecidos em função das condições de educação científica encontradas no CEADS. O estímulo para o desenvolvimento de novos currículos é observado pela criação de disciplinas como: Astronomia e Navegação, Diversidade Animal, Biodiversidade Vegetal, Ecofisiologia Vegetal, Ecologia de Rios e Córregos Tropicais, Expedições Científicas em Ecologia, Gestão Ambiental, História das Prisões, Introdução à Ecologia Marinha, Qualidade de Água em Estuário entre outras.

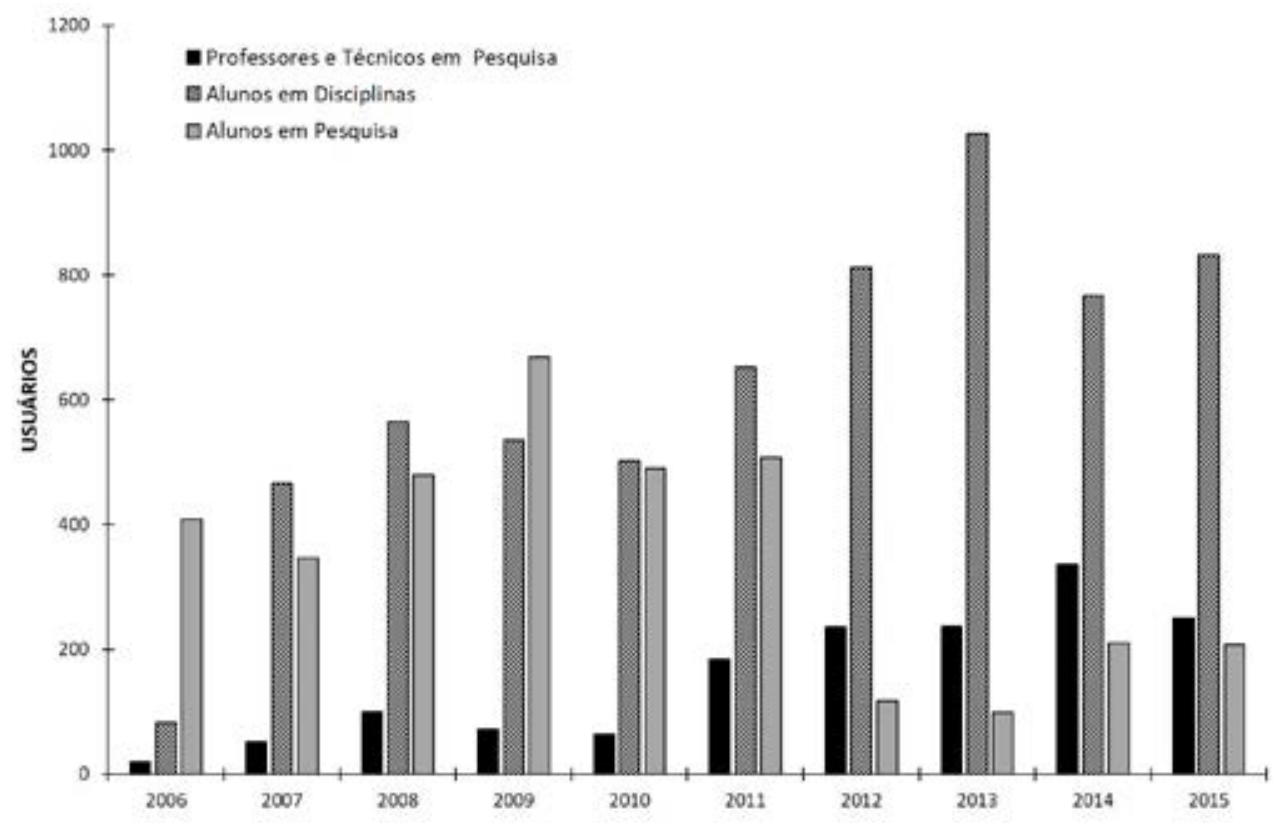

Figura 3. Número e perfil de usuários em expedição no CEADS no período de 2006 a 2015. 


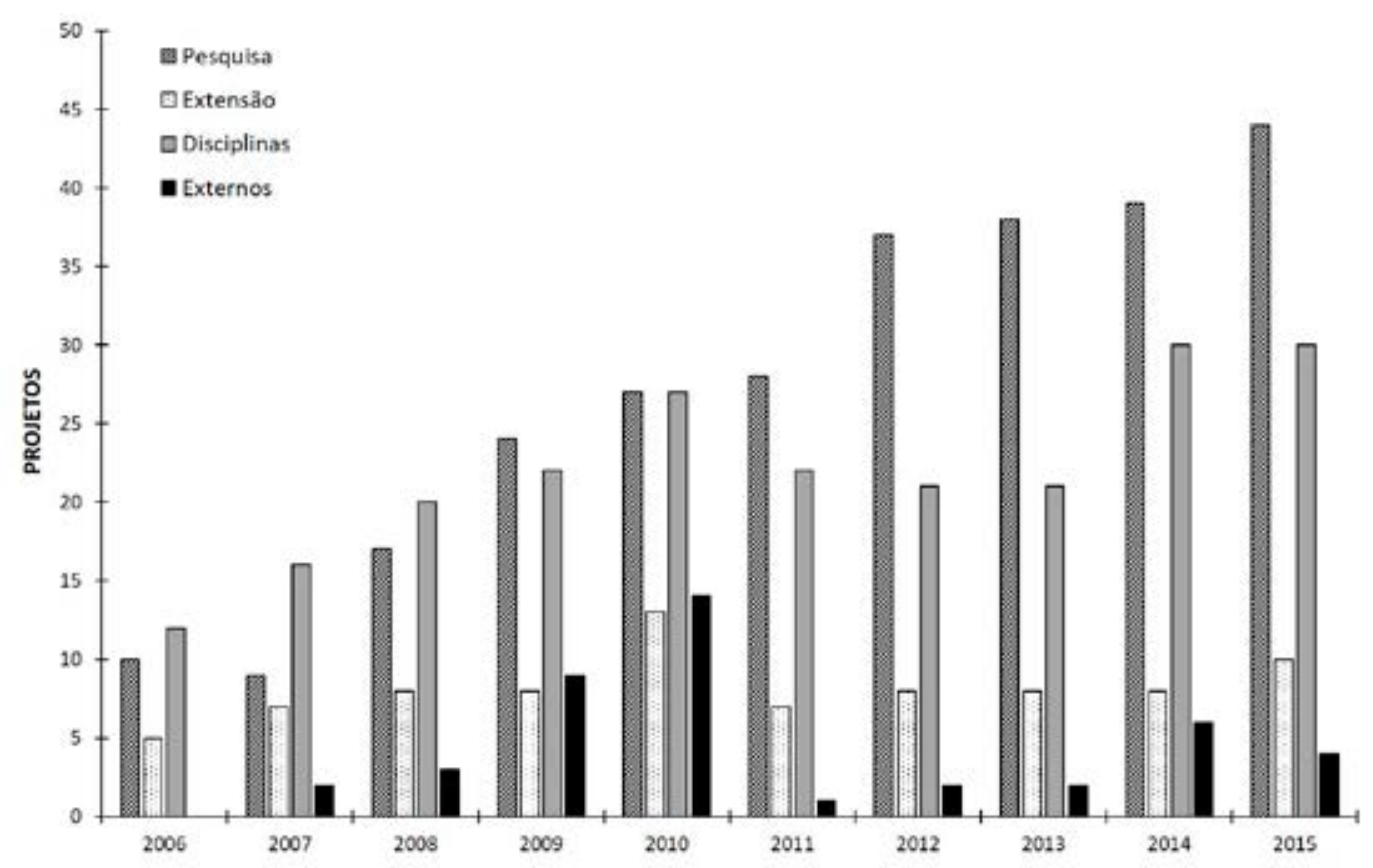

Figura 4. Número e natureza dos projetos desenvolvidos no CEADS no período de 2006 a 2015. Externos: projetos de outras Instituições de ensino, pesquisa e/ou extensão do estado do Rio de Janeiro ou de outros estados brasileiros.

A ampliação da abrangência do CEADS promoveu a nucleação de um novo departamento da UERJ, o Ecomuseu Ilha Grande, voltado à preservação da memória e do ambiente e, ainda, permitiu capitanear projetos para a construção do primeiro navio universitário do estado do Rio de Janeiro, cujos recursos financeiros foram adquiridos em parceria com diferentes Unidades Acadêmicas da UERJ.

A figura 4 evidencia a evolução do número e tipo de projetos cadastrados no CEADS entre 2006 e 2015. 140 projetos foram desenvolvidos nos últimos 10 anos de atividade. Embora a média de projetos com atividade regular seja 60 por ano, houve um aumento de quase $40 \%$ do número de projetos em ação nos dois últimos anos investigados (83 em ação em 2014 e 88, em 2015). Destes, 44\% são projetos de pesquisa, 17\% de extensão e 39\% disciplinas. Os projetos de outras Instituições de Ensino e/ou Pesquisa representam cerca de $7 \%$ dos projetos cadastrados no CEADS e revelam a importância deste Centro nos contextos acadêmicos fluminense e brasileiro.

Dentre as 34 Unidades Acadêmicas da UERJ com projetos cadastrados no CEADS, os departamentos que se destacaram em número de projetos são: Departamento de Ecologia (20 projetos); seguido dos Departamentos de Zoologia (12), de Biologia Vegetal (11), de Oceanografia Biológica (11) e de Ciências Biológicas da Faculdade de Formação de Professores - FFP (10). Tal proporcionalidade se mantém quando os departamentos que utilizam o CEADS são analisados por tipo de projeto executado: Departamento de Ecologia (11 projetos de pesquisa), Departamento de Biologia Vegetal (6), Departamento de Zoologia (5) e Departamentos de Oceanografia Biológica e de Ciências Biológicas - FFP (4 cada um). Quinze departamentos realizaram projetos de extensão no CEADS, com o número de projetos variando entre 1 e 3 por departamento. Quanto aos departamentos 
da UERJ que atuam com maior número de disciplinas no CEADS, destacaram-se: Departamentos de Ecologia e de Zoologia (7 disciplinas cada um) e Departamentos de Biologia Vegetal e de Ciências Biológicas da FFP (ambos com 6).

A partir de 2008, o número médio de usuários do CEADS em expedições de pesquisa passou a 500/ano. As equipes, formadas por professores, técnicos, alunos de pós-graduação e alunos de iniciação científica, realizaram as etapas de amostragem, instalação e monitoramento de experimentos in situ e utilizam a infraestrutura de hospedagem e laboratorial para a triagem do material investigado e processamento e análise parcial ou completa do mesmo. Os pesquisadores também dispuseram de infraestrutura de informação composta por biblioteca e acesso à Internet e realizaram reuniões para apresentação, debate, avaliação e informação de temas críticos para região, sempre ocupando uma posição de liderança com as Instituições participantes e fortalecendo o nome da UERJ, em âmbito brasileiro e internacional. Os resultados das pesquisas realizadas utilizando a infraestrutura do CEADS entre 2006 e 2015 propiciaram a elaboração de quase 800 produções científicas divididas na publicação de artigos científicos, livros, capítulos de livro e trabalhos completos em anais de congressos; e na conclusão de trabalhos de pós-graduação (mestrado e doutorado), de iniciações científicas e de monografias de conclusão de curso. Cabe destacar, que as pesquisas com base no CEADS já permitiram a descrição de táxons até então desconhecidos para ciência como: a cianobactéria Neolyngbya irregularis T.A. Caires, C.L. Sant'Anna et J.M.C. ${ }^{9}$; o parasita acantocéfalo, Anuracanthorhynchus tritaxisentis Bursey, Vrcibradic, Hatano \& Rocha ${ }^{10}$ os ácaros, Androlaelaps marmosops Hatano, Gettinger \& Bergallo ${ }^{11}$ e Tur megistoproctu Gettinger \& Bergalo ${ }^{12}$; os anuros Hylodes fredi Canedo \& Pombal J ${ }^{13}$ e Proceratophrys tupinamba Prado \& Pombal $\mathrm{Jr}^{14}$; e o roedor Rhipidomys itoan Costa, Geise, Pereira \& Costa ${ }^{15}$. Além da redescoberta de uma espécie arbórea endêmica e ameaçada de extinção Miconia gigantea Cogn. com mais de 100 anos sem registro de ocorrência in situ' ${ }^{16}$.

A análise do potencial do CEADS em redes de pesquisas tendo por base as publicações dos coordenadores de projetos revelou que CP1 apresentou 22 colaborações-articulações entre pesquisadores, CP2 apresentou 69 e CP3 apresentou 130 (Figuras 5 A-C). A abrangência das redes estabelecidas revelou cooperações intra e interdepartamentais, institucionais, nacionais e internacionais. CP2 apresentou colaborações em todos os níveis hierárquicos estabelecidos. Tanto $C P 1$ quanto $C P 3$ não apresentaram publicações no nível hierárquico alunos de graduação $(G)$. Este fato pode estar relacionado a conclusão das pesquisas iniciadas na graduação somente na pós-graduação $(P)$, como se verifica com as colaborações neste último nível hierárquico (CP1= 4 e CP3= 15 colaborações-articulações com alunos de pós-graduação). De acordo com o esperado, CP3 apresentou o maior número de colaborações-articulações internacionais (It). O maior número de colaborações-articulações de CP1 e CP2 foram estabelecidas com pesquisadores de outras instituições no Rio de Janeiro (RJ), enquanto CP3 apresentou o maior número de colaborações-articulações com pesquisadores de outras instituições no Brasil (B). Os três CPs apresentaram colaborações-articulações no seu próprio departamento (D), todavia apenas CP2 e CP3 estabeleceram colaborações-articulações com outras unidades acadêmicas dentro de sua própria Universidade $(I s=11, U=13$ e Is $=6, U=3$, respectivamente). Cabe destacar que são vários os fatores que levam os pesquisadores a colaborarem entre si. Entre esses se destacam: orientações, fornecimento do material de estudo, realização de alguma operação de rotina, uso coletivo de dados ou ideias, execução de maneira separada de diferentes partes de um projeto, necessidade de compartilhar o uso 
de equipamentos cada vez mais complexos e caros e novos padrões de financiamento adotados pelas agências de fomento, que visam potencializar o uso de recursos, evitar a duplicação de informações e aumentar a gama de instituições e pesquisadores beneficiados $^{17,18}$. Todos esses fatores podem ser constatados nas redes estabelecidas pelos três níveis de coordenadores de projetos.
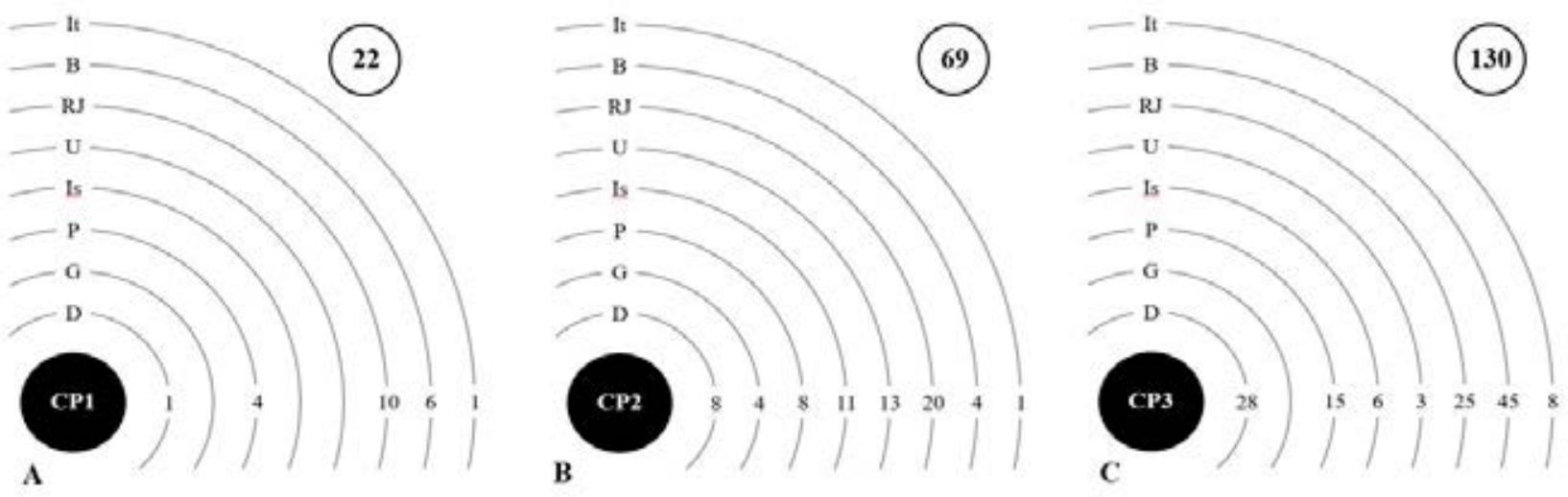

Figura 5. Colaboração-articulação de coordenadores de projetos (CP) em número e níveis hierárquicos. A: Coordenador de projetos no CEADS com pequena colaboração-articulação científica (CP1). B: Coordenador de projetos no CEADS com média colaboração-articulação científica (CP2). C: Coordenador de projetos no CEADS com grande colaboração-articulação científica (CP3). D: Pesquisadores do próprio Departamento. G: Alunos de Graduação. P: Alunos de Pós-graduação. Is: Pesquisadores do próprio Instituto. U: Pesquisadores da própria Universidade. RJ: Pesquisadores de outra Instituição no Rio de Janeiro. B: Pesquisadores de outra Instituição no Brasil. It: Pesquisadores de Instituições de outros países.

É importante ressaltar ainda que os centros de pesquisa diferem dos abrigos, casas ou pousadas de pesquisadores, que são os mais comumente encontrados no Brasil. Os abrigos são encontrados em várias Unidades de Conservação e áreas de interesse científico. De modo geral, nesses abrigos são oferecidos leitos, local para banho e elaboração de alimentos, alguns podem apresentar mais recursos em infraestrutura que outros, porém em número que raramente ultrapassa 15 usuários e sem o oferecimento de equipamentos para desenvolvimento de pesquisas ou tecnologias. São exemplos brasileiros nesse sentido, os abrigos do Parque Nacional do Itatiaia, do Parque Nacional da Serra dos Órgãos e do Parque Estadual da Ilha Grande, todos localizados no estado do Rio de Janeiro. Cabe destacar que seus usuários não fazem parte do corpo técnico das instituições que os oferecem. Casas ou pousadas para pesquisadores também são encontradas em várias instituições de ensino e pesquisa no Brasil. Nessa categoria de instalação, de modo geral, são oferecidos leitos, local para banho e alimentação, alguns apresentam vários recursos em infraestrutura e inerentes ao serviço hoteleiro, podendo abrigar um número variado de usuários, mas sem o oferecimento de equipamentos para desenvolvimento de pesquisas ou tecnologias, são exemplos: a Casa do Professor da Universidade Federal de Ouro Preto, Pousada do Pesquisador do Instituto de Pesquisas Jardim Botânico do 
Rio de Janeiro e Casa do Professor Visitante da UNICAMP. É importante notar que os usuários também não fazem parte do corpo técnico das instituições que oferecem estes tipos de instalação e as mesmas, normalmente, não estão localizadas em sítios de coleta, mas sim dentro das próprias instituições que as oferecem. Os Centros de Pesquisa, por sua vez, estão voltados à constituição de um espaço articulado entre produção de conhecimento e/ou tecnologias, formação e difusão, com trânsitos e trocas entre o saber fazer da instituição, os dados, as informações e as pesquisas, bem como as temáticas permanentes transversais e emergentes envolvendo a ciência. Seu intuito é identificar, desenvolver e apoiar a pesquisa e desenvolvimento de projetos que envolvam tecnologias avançadas dentro de uma vasta gama de setores. Nesses centros, são encontradas, além das condições oferecidas nos abrigos, casas ou pousadas para pesquisadores, a infraestrutura para pesquisas, contando com transporte, equipamentos, materiais de consumo e permanente e apoio técnico. Geralmente apresentam salas e auditórios para aulas, exposição de trabalhos e discussão de grupos em trabalho. Os usuários podem ou não fazer parte do corpo técnico das Instituições que oferecem este tipo de instalação. O número de pessoas é bem variado, sendo capaz de comportar turmas completas de alunos, são exemplos brasileiros nesta categoria: Centro de Estudos Ambientais da Universidade Estadual Paulista, Centro de Pesquisas Global da General Eletric no Brasil e Instituto de Desenvolvimento Sustentável Mamirauá.

\section{Conclusões}

Diante das questões inicialmente postuladas, observa-se que o CEADS atende a todas as diretrizes estabelecidas para a criação ou manutenção de um centro de pesquisa universitário.

As ações do CEADS são criticamente importantes para a projeção da Universidade do Estado do Rio de Janeiro no cenário da pesquisa, extensão e formação de recursos humanos, tendo contribuído para o fortalecimento da marca da UERJ e para que a Universidade ocupe uma posição de liderança entre Instituições congêneres. Essas ações também permitiram levantar fundos para novos investimentos no próprio Centro de Pesquisa, apoiando também os laboratórios envolvidos com as pesquisas desenvolvidas e que não estão fisicamente localizados no CEADS. Este Centro efetivamente contribui para formação de excelência de alunos e estimula o desenvolvimento de novos currículos, promovendo o ensino contextualizado e uma visão global e interdisciplinar que associa o ensino formal, não-formal e informal, onde o aluno é participante ativo, fazendo e envolvendo-se na situação da experiência. A influência do CEADS sobre outras instituições e setores, além daqueles que participam de sua própria iniciativa, sejam eles nacionais ou internacionais, pode ser aferida pelo crescente número de parcerias estabelecidas, pela utilização da infraestrutura existente por outras Instituições e pela representação da UERJ nos diferentes conselhos consultivos e deliberativos existentes na região sul do estado do Rio de Janeiro.

Cabe destacar também que em consonância com as perspectivas para as universidades do futuro ${ }^{19}$, o CEADS, além de ser fundamentalmente interdisciplinar em sua abordagem para pesquisa, ensino e extensão, abre espaço para participação de diferentes segmentos da sociedade, que opinam nas mais diversas questões de interesse regional e, embora pertencente a uma universidade pública, está em consonância com as demandas econômicas da região como pode ser observado pelas pesquisas em tecnologia e serviços nas áreas de 
maricultura, turismo e produção biotecnológica dos recursos naturais.

Embora as métricas de citação das publicações dos Coordenadores de Pesquisa do CEADS não tenham sido inventariadas, o relatório elaborado pela Clarivate Analytics ${ }^{20}$, com o panorama da produção científica do Brasil, revelou que o Brasil é o 13으 maior produtor de publicações de pesquisa em nível mundial e a Universidade do Estado do Rio de Janeiro apresenta o mais alto impacto de citação entre as universidades brasileiras. A UERJ também apresenta as maiores taxas de colaboração internacional, principalmente no que envolve as áreas da medicina, ciências da vida, matemática, ciências físicas, engenharia, tecnologia, ciências da terra, ciências do ambiente e ciências sociais.

Por fim, é importante notar que a Universidade do Estado do Rio de Janeiro por meio do CEADS contribui para melhoria das condições socioeconomicas e ambientais sem abandonar sua função de espaço de discussão, análise e crítica dos assuntos atuais. Essa vocação crítica que se estabelece necessariamente com liberdade e autonomia, o que é vital para uma universidade pluricultural, diversificada e sempre revolucionária.

\section{Agradecimentos}

Ao Staff administrativo do Centro de Estudos Ambientais e Desenvolvimento Sustentável pela disponibilização de dados; ao Dr. Marcelo Vianna Dias Machado Vianna Filho pela confecção do mapa e aos órgãos de fomento CNPq e FAPERJ pelas bolsas de pesquisa e apoio financeiro.

\section{Referências}

1. ALFARO, Y. La (re) orientación de la política de educación superior, investigación e innovación en el contexto del buen vivir. Universidades, v.69, p. 75-84, 2016.

2.PIMENTEL; T. D.; CARVALHO; F. C. C.; OLIVEIRA; M. C. B. Centros de Pesquisa em Turismo no Mercosul: distribuição, ênfases e possíveis interações como fator de desenvolvimento. Revista Gestão Universitária na América Latina - GUAL, v.11, 2018, p. 321-344.

3.SCHWARTZMAN, S. As universidades latino-americanas e sua contribuição para o desenvolvimento sustentável da região. In: SCHWARTZMAN, S. (Org.). Universidades e Desenvolvimento na América Latina - Experiências Exitosas de Centros de Pesquisas. 1ed Brasilia: Centro Edelstein de Pesquisas Sociais, v.1, 2008, p. 13-30.

4.SCHWARTZMAN, S. Pesquisa Universitária e Inovação no Brasil. In: Centro de Gestão e Estudos Estratégicos (Org.). Avaliação de políticas de ciência, tecnologia e inovação: diálogo entre experiências internacionais e brasileiras. 1 ed. Brasilia: Centro de Gestão e Estudos Estratégicos, v.1, 2008b, p. 19-43.

5.BASTOS, M. P.; PRADO, R. M.; SANTIAGO, A. M.; BIRMAN, P.; CATÃO, H.; MENDONÇA, T.; BAKKER, A.; FERRAREZ, A.; GILAYN, H.; MENDONCA, M.; WIEDEMANN, M.; ZANATTA, R.; PEREIRA, V.; CRUZ, A.; ROSEIRO, T.; ARAUJO, A. Estrutura econômica e organização sócio-cultural e política. In: BASTOS, M. P.; CALLADO C. H. (Orgs.) O Ambiente da llha Grande. 1ed. Rio de Janeiro: EDUERJ, v.1, 2009. p. 339-414.

6.CALLADO, C. H.; BARROS, A. A. M.; RIBAS, L. A.; ALBARELLO, N.; GAGLIARDI, R. F.; JASCONE, C. E. Flora e cobertura vegetal. In: BASTOS, M. P.; CALLADO, C. H. (Orgs.) O Ambiente da llha Grande. 1ed. Rio de Janeiro: EDUERJ, v.1, 2009, p. 91-162.

7. CHAIMOVICH, H. Apresentação. In: SCHWARTZMAN, S. (Org.). Universidades e Desenvolvimento na América Latina - Experiências Exitosas de Centros de Pesquisas. 
1ed. Brasília: Centro Edelstein de Pesquisas Sociais, v.1, 2008, p. 4-8.

8.UNESCO. A Ciência no século XXI: uma nova missão e uma base de ação. UNESCO, Brasília, v.1, 2003, p. 72.

9.CAIRES, T. A.; LYR G. M.; HENTSCHKE, G. S.; PEDRINI A. G.; SANT'ANNA, C. L.; NUNES, J. M. C. Neolyngbya gen. nov. (Cyanobacteria, Oscillatoriaceae): a new filamentous benthic marine taxon widely distributed along the Brazilian coast. Molecular Phylogenetics and Evolution, v.120, p. 196-211, 2018.

10.BURSEY, C. R.; VRCIBRADIC, D.; HATANO, F. H.; ROCHA, C. F. D. New genus, new species of Acanthocephala (Echinorhynchidae) from the Brazilian Frog Hylodes phyllodes (Anura: Leptodactylidae). Journal of Parasitology, v.92, p. 353-356, 2006.

11. HATANO, F. H.; GETTINGER, D.; VAN SLUYS, M.; ROCHA, C. F. D. Parasitism in Hylodes phyllodes (Anura: Cycloramphidae) by Hannemania sp. (Acari: Trombiculidae) in an area of Atlantic Rainforest, Ilha Grande Southesatern Brazil. Parasite, v.14, p. 107$108,2007$.

12.GETTINGER, D.; BERGALLO, H. G. A new species of Laelapine mite (Acari: Parasitiformes: Laelapidae) associated with Proechimys dimidiatus in the Atlantic forests of Brazil. Journal of Parasitology, v.89, p. 705-708, 2003.

13.CANEDO, C.; POMBAL Jr. J. P. Two new species of torrent frog of the genus Hylodes (Anura, Hylodidae) with nuptial thumb tubercles. Herpetologica, v.63, p. 224-235, 2007.

14.PRADO, G. M.; POMBAL Jr, J. P. Espécies de Procerathophrys Miranda-Ribeiro, 1920 com apêndices palpebrais (Anura: Cycloramphidae). Arquivos de Zoologia, v.39, p. 1-85, 2008.

15.COSTA, B. M. A.; GEISE, L.; PEREIRA, L. G.; COSTA, L. P. Phylogeography of Rhipidomys (Rodentia: Cricetidae: Sigmodontinae) and description of two new species from southeastern Brazil. Journal of Mammalogy, V.92, p. 945-962, 2011.

16.ROSA, L. P. G.; BAUMGRATZ, J. F. A.; SILVA NETO, S. J. Miconia gigantea, a long-forgotten endemic and endangered species of Melastomataceae in the Brazilian Atlantic Forest. Anais da Academia Brasileira de Ciências, v.88, p. 1809-1818, 2016.

17.SUBRAMANYAM, K. Bibliometric studies of research collaboration: a review. Journal of Information Science, v.6, p. 33-38, 1983.

18.BALANCIERI, R.; BOVO, A. B.; KERN, V. M.; PACHECO, R. C. S.; BARCIA, R. M. A análise de redes de colaboração científica sob as novas tecnologias de informação e comunicação: um estudo na Plataforma Lattes. Ciência da Informação, v.34, p. 64-77, 2005.

19.NATURE. The University of the Future. Nature, v.446, p. 949, 2007.

20.CROSS, D. I.; THOMSON, S.; SIBCLAIR, A. Research in Brazil: a report for CAPES by Clarivate Analytics, 2018. [Viewed 08 February 2018] Available form: http://www.capes. gov.br/images/stories/download/diversos/17012018-CAPES-InCitesReport-Final.pdf 